Monatsschr Kinderheilkd 2018 • 166:774-784 https://doi.org/10.1007/s00112-018-0525-z Online publiziert: 15. Juni 2018

(c) Der/die Autor(en) 2018

\section{Redaktion}

R. Kerbl, Leoben

K. Schmitt, Linz

CrossMark

\author{
S. B. Wortmann ${ }^{1,2,3} \cdot$ H.-C. Duba ${ }^{4}$ \\ ${ }^{1}$ Kinderklinik, Salzburger Landeskliniken (SALK), Salzburg, Österreich \\ ${ }^{2}$ Paracelsus Medizinische Privatuniversität, Salzburg, Österreich \\ ${ }^{3}$ Institut für Humangenetik, Klinikum rechts der Isar, Technische Universität München, München, \\ Deutschland \\ ${ }^{4}$ Institut für Medizinische Genetik, Med Campus IV, Kepler Universitätsklinikum, Linz, Österreich
}

\title{
Angewandte Genetik in der Pädiatrie
}

\begin{abstract}
Die Pädiatrie ist das breiteste und dynamischste Fachgebiet der gesamten Medizin. Im Mittelpunkt steht das Kind mit seiner Familie von der Geburt bis zum Erwachsenenalter. Mehr als in anderen Fachgebieten spielen angeborene Erkrankungen eine Rolle. Die zunehmende Verfügbarkeit der "Next Generation Sequencing"(NGS)-Methoden in den letzten 5 Jahren zeigt, dass die meisten dieser Erkrankungen genetischen bzw. monogenen Ursprungs sind, und eröffnet ungeahnte Möglichkeiten, aber auch Herausforderungen in Diagnostik und Therapie.
\end{abstract}

\section{Hintergrund}

\section{Bedeutung genetischer Erkrankungen in der Pädiatrie}

Die Bedeutung genetischer bzw. monogener Erkrankungen zeigt sich bereits weit vor der Geburt eines Kindes, da genetische Erkrankungen die häufigste Ursache für Fehlgeburten sind. Studien ergeben, dass ca. $30 \%$ aller Konzeptionen vor der Implantation und weitere $30 \%$ zwischen Implantation und der 4. Schwangerschaftswoche verloren gehen [14]. Untersuchungen der zugrunde liegenden Ursachen beschreiben schwere genetische Erkrankungen in den betroffenen Feten, die mit dem extrauterinen Leben nicht vereinbar sind, wie z. B. numerische Chromosomenaberrationen (Aneuploidie, [3]). Monogenetische Erkrankungen werden ebenfalls als Ursache für Spätaborte beschrieben (z. B. Meckel-
Gruber-Syndrom, Osteogenesis imperfecta Typ II), und auch in Totgeburten sind angeborene Fehlbildungen häufig. So finden sich beispielweise bei $10 \%$ der Betroffenen Herzfehler [10].

\section{》) Genetische bzw. monogene Erkrankungen verursachen $35 \%$ aller Todesfälle bei Säuglingen}

Der Facharzt für Kinder- und Jugendmedizin/Jugendheilkunde begegnet Kindern mit genetischen bzw. monogenen Erkrankungen in allen Altersstufen, allerdings ist die Erstpräsentation häufig in der Neugeborenenperiode (z. B. angeborene Stoffwechselerkrankungen, Herzfehler im Rahmen von syndromalen Erkrankungen wie Trisomie 21) oder im Kleinkindalter (z. B. genetisch bedingte Entwicklungsverzögerung). Generell zählen genetische bzw. monogene Erkrankungen zu den der häufigsten Gründen für pädiatrische Krankenhausaufenthalte und verursachen $35 \%$ aller Todesfälle bei Kindern im Alter unter einem Jahr [4].

Die meisten der pädiatrischen genetischen bzw. monogenen Erkrankungen sind selten und treten definitionsgemäß bei weniger als einem von 2000 Menschen auf. Auch wenn sie aufgrund einer häufig damit verbundenen eingeschränkten Reproduktionsfähigkeit in der Gesamtbevölkerung einen nur geringen Anteil darstellen, betreffen sie in ihrer Gesamtheit dennoch viele Kinder und unterstreichen damit die Bedeutung genetischer Erkrankungen in der täglichen pädiatri- schen Patientenversorgung. Der zunehmende Stellenwert genetischer Untersuchungen in der Pädiatrie impliziert, dass das Wissen darüber eine entscheidende Voraussetzung für die Tätigkeit des Facharztes für Kinder- und Jugendmedizin/Jugendheilkunde geworden ist. Immer dann, wenn sich Anhaltspunkte für eine genetisch bedingte Erkrankung ergeben, haben Betroffene bzw. seine Sorgeberechtigen das Recht, über die Möglichkeit der genetischen Beratung und Diagnostik aufgeklärt zu werden. Sie ist Teil der Krankenversorgung, auch wenn die Kostenübernahme für die NGS-Methoden durch die Krankenversicherungen nicht immer gewährleistet ist.

\section{Formen genetischer Erkrankungen und Vererbungsmodi}

Das menschliche Genom umfasst ca. 20.000 Gene, die sich auf 22 Chromosomen (Autosomen) und die $2 \mathrm{Ge}$ schlechtschromosomen des Zellkerns sowie die in hoher Kopienanzahl vorliegende ringförmige DNA der Mitochondrien verteilen. Die Ursachen genetischer Erkrankungen rangieren von der Substitution, Deletion oder Duplikation eines einzelnen Basenpaars („single nucleotide variants", SNV) in einzelnen Genen (monogene Erkrankungen) über die veränderte Kopienzahl („copy number variations", $\mathrm{CNV}$ ) kleinerer oder größerer Chromosomenabschnitte bis hin zu veränderten Chromosomenanzahlen (z.B. Trisomie 21). Neben diesen eher „einfachen“ strukturellen Veränderungen gibt es auch kompli- 
Hier steht eine Anzeige.

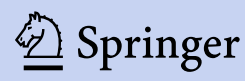


Tab. 1 Genetische Routinemethoden

\begin{tabular}{|c|c|c|c|c|c|c|c|}
\hline & $\begin{array}{l}\text { Lichtmikro- } \\
\text { skopische } \\
\text { Chromosomen- } \\
\text { analyse }\end{array}$ & $\begin{array}{l}\text { Karyo- } \\
\text { typisierung }\end{array}$ & Microarray & $\begin{array}{l}\text { Einzelgen- } \\
\text { analyse }\end{array}$ & Gen-Panel & WES & WGS \\
\hline Auflösung & $\begin{array}{l}\text { Gesamtes Chro- } \\
\text { mosom }\end{array}$ & 5-15 Mbp & 50-100 Mbp & $1 \mathrm{bp}$ & $1 \mathrm{bp}$ & $1 \mathrm{bp}$ & $1 \mathrm{bp}$ \\
\hline $\begin{array}{l}\text { Anzahl getesteter } \\
\text { Loci }\end{array}$ & - & Сa. 500 & 0,05-2 Mio. & 1 & $50-1000$ & 20.000 & $\geq 20.000$ \\
\hline $\begin{array}{l}\text { Art gefundener } \\
\text { Varianten }\end{array}$ & $\begin{array}{l}\text { Aneuploidie, } \\
\text { Polyploidie }\end{array}$ & $\begin{array}{l}\text { Varianten } \\
>5 \mathrm{Mbp}\end{array}$ & $\begin{array}{l}\text { „Copy number } \\
\text { variations“ (CNV) }\end{array}$ & $\begin{array}{l}\text { Punktmuta- } \\
\text { tionen, kleine } \\
\text { Insertionen } \\
\text { bzw. Dele- } \\
\text { tionen }\end{array}$ & $\begin{array}{l}\text { Varianten in } \\
\text { selektierten } \\
\text { Krankheitsge- } \\
\text { nen }\end{array}$ & $\begin{array}{l}\text { Varianten in } \\
\text { codierenden } \\
\text { Regionen }\end{array}$ & $\begin{array}{l}\text { Mehrheit al- } \\
\text { ler Varianten }\end{array}$ \\
\hline $\begin{array}{l}\text { Anzahl gefundener } \\
\text { Varianten/Person }\end{array}$ & 0 oder 1 & 0 oder 1 & $\begin{array}{l}10 \text { bis mehre- } \\
\text { re } 100\end{array}$ & 0 oder 10 & $\begin{array}{l}10 \text { bis mehre- } \\
\text { re } 100\end{array}$ & Сa. 30.000 & 4-5 Mio. \\
\hline $\begin{array}{l}\text { Diagnostische } \\
\text { Ausbeute }\end{array}$ & Niedrig & - & - & $\begin{array}{l}\text { Abhängig von } \\
\text { Verdachtsdia- } \\
\text { gnose }\end{array}$ & - & - & Hoch \\
\hline
\end{tabular}

ziertere genetische Veränderungen wie z.B. intragenische Duplikationen (z.B. Rett-Syndrom), intragenische Expansionen von Basentripletts (z.B. fragiles $\mathrm{X}$-Syndrom, Friedreich-Ataxie, myotone Dystrophie) oder uniparenterale Disomien (beide Chromosomen eines homologen Chromosomenpaars stammen von einem Elternteil, z. B. PraderWilli-/Angelmann-Syndrom). Eine weitere Besonderheit stellen Mutationen in der mitochondrialen DNA (mtDNA) dar. In einer Zelle können Kopien mit und ohne Mutationen vorliegen (Heteroplasmie). Erst, wenn ein bestimmter Schwellenwert überschritten wird und ein hoher Anteil von mutierten mtDNAKopien vorliegt, kommt es zum mitochondrialen Funktionsverlust und zum Auftreten von Krankheitssymptomen.

In der Regel liegen alle genetischen Informationen bzw. ihre Veränderung in allen Körperzellen vor, allerdings kann sich die Veränderung auch nur in einem Teil der Zellen finden (genetisches Mosaik, z.B. Turner-Syndrom, epileptische Enzephalopathie [25]). Genetische Veränderungen können u.U. nur im betroffenen Gewebe (z. B. im Muskel bei mitochondrialer Myopathie), nicht aber im Blut (korrekter der Leukozyten-DNA) vorliegen. Letzteres gilt auch für Mutationen in Tumoren, wobei auf dieses Thema im vorliegenden Beitrag nicht näher eingegangen wird. Bei weiblichen Patienten ist außerdem die X-Chromosom-Inaktivierung $\mathrm{zu}$ bedenken. Bei ungleicher (Skewed-)X-Chromosom-Inaktivierung wird immer das gesunde X-Chromosom jeder Zelle deaktiviert und so können auch bei weiblichen Patienten X-Chromosom-gebundene Erkrankungen auftreten (z.B. Pyruvat-DehydrogenaseKomplex[PDHc]-Defizienz aufgrund von PDHA1-Mutationen).

Genetische Erkrankungen können vererbt werden (autosomal-dominant, autosomal-rezessiv, X-Chromosom-gebunden, maternal etc.), oder die Veränderungen können im Betroffenen neu entstehen (de novo). Die NGS-Techniken revolutionieren gerade viele grundsätzliche Einsichten in die menschliche Genetik. Zum Beispiel konnte durch Sequenzierung der DNA-Proben von Eltern-KindTrios gezeigt werden, dass genetischen Mutationen, die einer Intelligenzminderung/Entwicklungsverzögerung oder einer Epilepsie zugrunde liegen, häufig autosomal-dominante Neumutationen (de novo) sind. Das erklärt auch, weshalb diese Erkrankungen sich trotz oft stark reduzierter Fertilität einer evolutionären Selektion widersetzt haben $[22,30]$.

Aktuell (Stand 07.04.2018) sind in der Datenbank Online Mendelian Inheritance in Man (OMIM, www.omim. org) 5222 Krankheitsbilder aufgeführt, für die eine molekulargenetische Basis als bekannt angenommen werden kann.
Davon folgen 4863 einem autosomaldominanten oder autosomal-rezessiven Erbgang, 324 werden X-Chromosomgebunden, 4 Y-Chromosom-gebunden und 31 mitochondrial vererbt. Monatlich wurden im Jahr 2017 durchschnittlich 47 neue „Gen-Krankheitsbild-Kombinationen“ in der OMIM-Datenbank eingetragen (https://www.omim.org/statistics/ update). Für 1584 Erkrankungen liegt eine phänotypische Beschreibung vor, mit jedoch unbekannter molekularer Basis; für weitere 1769 wird eine solche vermutet.

\section{Diagnostik}

\section{Klassische genetische Unter- suchungsmethoden}

Lange Zeit bestand die genetische Routinediagnostik aus 2 grundsätzlich gegensätzlichen Untersuchungsmethoden (• Tab. 1): zielgerichtete hochauflösende Einzelgenanalyse und niedrigauflösende zytogenetische Tests. In der Einzelgenanalyse wird, basierend auf der phänotypischen Analyse des untersuchenden Arztes, ein einzelnes Gen für die molekulargenetische Untersuchung mithilfe der Sanger-Sequenzierung ausgewählt und dann Base für Base untersucht. Die Wahrscheinlichkeit einer Diagnose beruht bei dieser Methode also gänzlich auf der Fähigkeit des Arztes, eine 
korrekte klinische Diagnose zu stellen und das richtige Zielgen auszuwählen. Daher sind Einzelgenanalysen sehr gut geeignet, um klinisch gut abgegrenzte Erkrankungen zu diagnostizieren, für die krankheitsverursachende Varianten nur in einem oder einigen wenigen Genen vorkommen. Beispiele hierfür sind zystische Fibrose (biallelische Varianten im CTFR-Gen) oder DuchenneMuskeldystrophie (monoallelische Varianten im X-Chromosom-gebundenen $D M D$-Gen). Den gegensätzlichen Ansatz verfolgt die Karyotypisierung (5bis15-Mbp-Auflösung), ein niedrigauflösender „whole genome approach“, der genutzt wird, um Monosomien, Trisomien oder andere große chromosomale Imbalancen zu diagnostizieren. Zur Entdeckung kleinerer CNV eignet sich die Methode der genomischen Microarrays ebenso (Auflösung ca. 50-100 kb). Hiermit lassen sich CNV an jeder Position im Genom entdecken, einschließlich der rekurrenten Varianten, assoziiert mit klassischen (z.B. Angelmann-, Williams-Beuren-, Smith-Magenis-Syndrom) oder neueren Mikrodeletions-/ Mikroduplikationssyndromen [29].

Mit diesem Ansatz ist die Aufklärungsrate jedoch niedrig, da zytogenetische Tests nur ca. $10 \%$ aller pädiatrischen Patienten mit Verdacht auf eine seltene genetische Erkrankung zu einer Diagnose führen [24].

\section{Moderne genetische Unter- suchungsmethoden}

\section{Prinzip}

Die NGS-Methoden haben die genetische Diagnostik in den letzten Jahren revolutioniert, da sie die gleichzeitige Sequenzierung sehr vieler Gene ermöglichen (• Tab. 1; [29]). Sie geben dem anfordernden Arzt die Wahl, mehrere Gene gleichzeitig (z.B. EpilepsiePanel) oder aber gleich das gesamte Exom (alle proteincodierenden [exomischen] Genabschnitte, „whole exome sequencing",WES) oder das gesamte Genom (die vollständige DNA-Sequenz, „whole genome sequencing“, WGS) des Patienten $\mathrm{zu}$ sequenzieren und nach krankheitsverursachenden Varianten zu suchen.
Diese Methoden eignen sich ausgezeichnet zur Abklärung diverser monogener Krankheitsbilder mit nichtunterscheidbaren, unspezifischen klinischen Phänotypen, denen Veränderungen in diversen Genen zugrunde liegen können. Beispiele hierfür sind mitochondriale Erkrankungen sowie unklare Intelligenzminderung oder Entwicklungsverzögerung und epileptische Enzephalopathie (• Tab. 3).

\section{》) Next generation sequencing ermöglicht gleichzeitige Sequenzierung sehr vieler Gene}

Die Suche in der OMIM-Datenbank mit dem Begriff „developmental delay“ liefert aktuell (April 2018) 969 Einträge für Krankheitsbilder, in deren phänotypischer Beschreibung der Begriff Entwicklungsverzögerung vorkommt und für die der zugrunde liegende genetische Defekt bekannt ist. Mithilfe der WES ist es möglich, SNV in den codierenden Bereichen (Exone) aller ca. 20.000 menschlichen Gene sowie der mtDNA zu finden. Ebenso können CNV jeglicher Größe entdeckt werden [21]. Die Frage, ob bei allen Kindern mit unklarer Entwicklungsverzögerung zunächst ein Array durchgeführt werden sollte, bevor auf eine WES oder WGS übergegangen wird, lässt sich nicht pauschal beantworten, sondern hängt von den exakten technischen Gegebenheiten der verwendeten Testmethoden und der Erfahrung des Labormitarbeiters und der Auswerters ab [21].

\section{Gen-Panel, Whole exome sequencing oder Whole genome sequencing}

Das oben Gesagte gilt für die Entscheidung zwischen Gen-Panel, WES oder WGS (• Abb. 1). Die Methoden unterscheiden sich bezüglich Anzahl der sequenzierten Zielregionen, Auflösung bzw. Sequenziertiefe, aktuellen Entwicklungen von Auswertealgorithmen und Preis für die Prozedur. Gen-Panels sequenzieren eine Auswahl bekannter Gene, in denen Varianten beschrieben sind, die ursächlich mit einer Erkrankung in Verbindung gebracht wurden (Krank-
Monatsschr Kinderheilkd 2018 · 166:774-784 https://doi.org/10.1007/s00112-018-0525-z ๑ Der/die Autor(en) 2018

\section{S. B. Wortmann - H.-C. Duba}

\section{Angewandte Genetik in der Pädiatrie}

\section{Zusammenfassung}

Mehr als in anderen Fachgebieten gehören angeborene Erkrankungen zum Alltag des Facharztes für Kinder- und Jugendmedizin. Die zunehmende Verfügbarkeit der Next Generation Sequencing-Methoden in den letzten 5 Jahren zeigt, dass die meisten dieser Erkrankungen genetischen bzw. monogenen Ursprungs sind, und eröffnet ungeahnte Möglichkeiten, aber auch Herausforderungen in Diagnostik und Therapie. Dieser Beitrag bietet einen Überblick über die Bedeutung genetischer Erkrankungen in der Pädiatrie, erläutert die Möglichkeiten, aber auch Limitationen moderner genetischer Diagnostik wie der Exomsequenzierung und ihre Auswirkungen auf die tägliche Patientenversorgung.

\section{Schlüsselwörter}

"High-throughput nucleotide sequencing" . "Whole exome sequencing" • "Whole genome sequencing" · Entwicklungsverzögerungsstörungen · Intelligenzminderung

\section{Applied genetics in pediatrics}

\section{Abstract}

Pediatricians significantly more often involved in the daily treatment of patients with congenital disorders than any other medical specialist. The increasing availability of next generation sequencing techniques during the last 5 years shows that most of these congenital disorders are of genetic resp. monogenic origin and offers tremendous opportunities but also challenges for diagnostics and treatment. This article provides an overview on the importance of genetic disorders in pediatrics, explains the advantages but also limitations of modern genetic techniques, such as exome sequencing and finally deals with the consequences for daily clinical care.

Keywords

High-throughput nucleotide sequencing . Whole exome sequencing . Whole genome sequencing - Developmental delay disorders - Mental retardation 


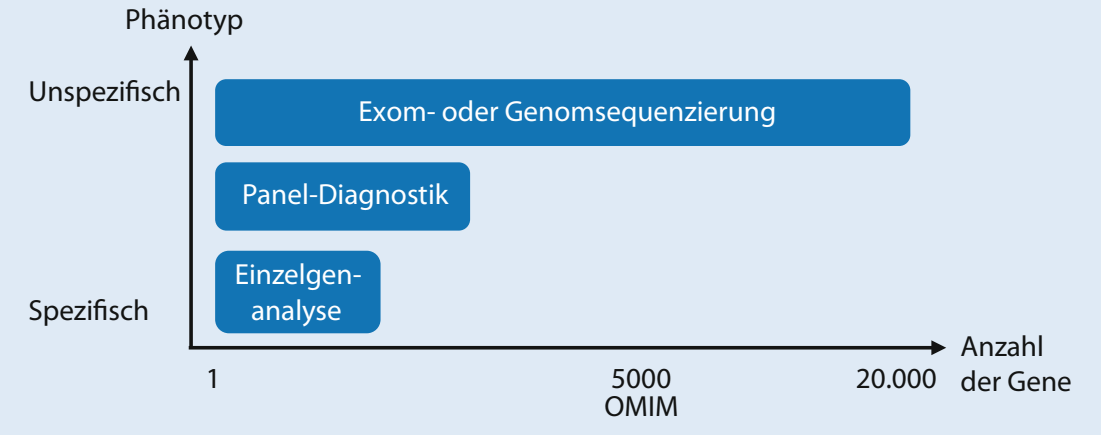

Abb. 1 A Anzahl der abgedeckten Krankheitsgene und sich daraus ergebende Einsatzmöglichkeiten für die genetischen Techniken bei Verdacht auf eine monogene Erkrankung

heitsgene), mit hoher Auflösung und garantieren eine gute Abdeckung aller Bereiche von Interesse. Sie sind insofern limitiert, da sie nur die Gene umfassen, die zum Zeitpunkt der Panel-Erstellung als Krankheitsgene bekannt waren. Betrachtet man, dass allein die „deciphering developmental disorders(DDD)-study“ 27 Gene erstmals mit Epilepsie assoziierte (als Krankheitsgen definierte), ist auch hier das Risiko groß, Diagnosen „zu verpassen“ [32]. Je unspezifischer der Phänotyp ist, desto größer ist meist die Anzahl der Krankheitsgene.

Mithilfe der WGS werden sowohl die Exon- als auch die Intron-, sowie intergene Abschnitte der menschlichen DNA untersucht. Da kein Anreicherungsschritt notwendig ist, ist die Datengenerierung schneller als bei der WES, die Herausforderung ist jedoch die damit verbundene Datenanalyse. Die $2 \times 3 \mathrm{Mrd}$. Basenpaare eines menschlichen Genoms (mütterliches und väterliches Genom) enthalten ca. 4-5 Mio. Varianten/Person. Mithilfe geeigneter Filter muss hieraus die krankheitsverursachende Mutation isoliert werden. Da die Exomsequenzierung momentan zeit- und kostengünstiger ist, hat sie sich bisher als Standardmethode etabliert.

\section{Whole exome sequencing} Grundlegende technische Aspekte. Die WES wird stets häufiger in der klinischen Routine eingesetzt, die Methode wurde ausführlich in der Monatsschrift Kinderheilkunde beschrieben [9]. Hierzu werden ca. 3-5 ml Ethylendiamintetraessigsäure(EDTA)-Blut (bei Säuglingen ge- nügen $2 \mathrm{ml}$ ) benötigt. Die DNA wird aus Leukozyten extrahiert und zerkleinert. Die fragmentierten DNA-Abschnitte, die codierende (Exon-)Sequenzen enthalten, werden an komplementäre Sequenzen gebunden. Die nichtcodierende IntronDNA wird ausgewaschen. Die angereicherten Exon-DNA-Fragmente werden vervielfältigt und parallel sequenziert. Nach Abschluss der Sequenzierung werden Millionen dieser „reads“ mit der Referenzsequenz verglichen und ihrem Platz im Genom zugordnet. Um Mutationen von technischen Artefakten (falsch positive Varianten) unterscheiden $\mathrm{zu}$ können, ist eine Mehrfachsequenzierung jeder Sequenzposition notwendig. Eine 20-fache Mindestabdeckung von 98-99\% aller Exone wird in der Regel angestrebt. Die schließlich vorliegende Konsensussequenz jeder einzelnen Sequenzposition dokumentiert Abweichungen von der Referenzsequenz. Die Abweichungen werden als Varianten bezeichnet. Im Mittel finden sich bei jedem Menschen ca. 30.000 Varianten in den Exons bzw. an den Exon-IntronGrenzen. Die Mehrzahl dieser Varianten ist nicht krankheitsrelevant; es bedarf daher einer Reihe von Filterschritten, um eine einzige pathogene Variante für eine monogene Erkrankung aufzurufen.

\section{Auswertung: von $\mathbf{3 0 . 0 0 0 ~ V a r i a n t e n ~ z u r ~}$} Mutation. WES-Daten werden weltweit nach ähnlichen Algorithmen ausgewertet.

Die Datenfilterung beruht dabei auf 2 Grundannahmen: Krankheitsverursachende Varianten führen zu Proteinver- änderungen und sind in der Gesamtbevölkerung selten. Hierzu werden alle Varianten mit verschiedenen Datenbanken (z.B. „single nucleotide polymorphism database" [dbSNP], hausinterne Datenbanken) abgeglichen. Eine entscheidende Rolle spielen die öffentlich zugänglichen Datenbanken Exome Aggregation Consortium (ExAC; exac.broadinstitute.org) und Genome Aggregation Database (GnomAD; gnomad.broadinstitute.org), die aktuell zusammen mehr als 180.000 WES-Datensätze und mehr als 15.000 WGS-Datensätze gesunder Kontrollpersonen enthalten. Durch Abgleich mit diesen Datenbanken können Varianten herausgefiltert werden, die in der gesunden Normalbevölkerung bisher nicht bzw. selten (Allelfrequenz $<1 \%$ bei autosomal rezessiven, $<0,1 \%$ bei autosomal dominanten Erkrankungen) vorkommen.

\section{„Klinische Exomanalyse"}

Im Rahmen der Datenanalyse wird auch überprüft, ob sich Varianten in bekannten OMIM-gelisteten Krankheitsgenen finden. Solche Daten sind in der ClinVar-Datenbank (www.ncbi.nlm.nih.gov/ clinvar/) und der Human-Gene Mutation Database (HGMD, www.gmd.cf. ac.uk/ac/index.php) abrufbar. Ist eine Variante hier als krankheitsauslösend beschrieben, wird sie als pathogen eingestuft und berichtet (• Tab. 2). Häufig findet sich nicht passgenau die betreffende Variante des Patienten, sondern eine bisher unbekannte Variante in einem Krankheitsgen. Dann wird mithilfe von Software-Programmen (SIFT [13], Polyphen [1], CADD [11]) eingeschätzt, ob die betreffende Variante die Proteinfunktion beeinträchtigt. Abhängig von der Einschätzung wird diese Variante als wahrscheinlich pathogen oder als Variante unklarer Signifikanz (VUS) berichtet. Durch ergänzende funktionelle Untersuchungen z.B. in Fibroblasten und/oder erneute/erweiterte Phänotypisierung des Patienten kann eventuell der Nachweis erbracht werden, dass es sich um eine krankheitsverursachende Variante als Mutation handelt.

Oben genannte Schritte werden sowohl unter Annahme eines autosomaldominanten, eines autosomal-rezessi- 


\begin{tabular}{|l|l|l}
\hline ACMG-Kategorie & Klassifikation & Beschreibung \\
\hline 5 & $\begin{array}{l}\text { Benigne (entsprechend "sehr geringe Wahr- } \\
\text { scheinlichkeit") }\end{array}$ & $\begin{array}{l}\text { Varianten in jeglichen Genen, die auch in der gesunden Gesamtbevölkerung } \\
\text { häufig vorkommen }\end{array}$ \\
\hline 4 & $\begin{array}{l}\text { Wahrscheinlich benigne (entsprechend "ge- } \\
\text { ringe Wahrscheinlichkeit") }\end{array}$ & $\begin{array}{l}\text { Beispielsweise synonyme Varianten in bekannten Krankheitsgenen, die weder } \\
\text { die Proteinstruktur noch das Spleißen beeinflussen oder Intron-Varianten }\end{array}$ \\
\hline $\begin{array}{l}\text { Variante unklarer Signifikanz (VUS) } \\
\text { Gruppe I: tendenziell benigne; Gruppe Il: ohne } \\
\text { Tendenz; Gruppe III: tendenziell pathogen }\end{array}$ & $\begin{array}{l}\text { Beispielweise Varianten, die die Proteinstruktur beeinflussen, wobei der Einfluss } \\
\text { auf die Proteinfunktion aber unsicher ist oder Varianten in einem Krankheits- } \\
\text { gen, das nicht zum Phänotyp des Patienten passt, oder Varianten in einem } \\
\text { Kandidatengen }\end{array}$ \\
\hline 2 & $\begin{array}{l}\text { Wahrscheinlich pathogen }(90-99 \%, \text { entspre- } \\
\text { chend "hohe Wahrscheinlichkeit") }\end{array}$ & $\begin{array}{l}\text { Nicht vorbeschriebene, aber wahrscheinlich pathogene Variante in einem be- } \\
\text { kannten Krankheitsgen }\end{array}$ \\
\hline 1 & $\begin{array}{l}\text { Pathogen (>99\%, entsprechend "sehr hohe } \\
\text { Wahrscheinlichkeit") }\end{array}$ & Als pathogen bekannte (publizierte) Variante in einem Krankheitsgen \\
\hline ACMG American College of Medical Genetics & \\
\hline
\end{tabular}

ven, eines X-Chromosom-gebundenen und eines mitochondrialen Erbgangs durchgeführt. Desweiteren erfolgt eine gezielte Analyse nach Sequenzduplikationen und Deletionen (CNV-Analyse). Falls möglich, wenn Eltern-Kind-TrioDaten vorliegen, wird eine de novo-Mutationssuche angeschlossen. Generell ist eine Trio-WES leichter auszuwerten, da die elterlichen Daten verfügbar sind und sofort sichtbar ist, ob z.B. 2 Varianten beim Kind auch tatsächlich biallelisch vorliegen. Dieser Zeitaspekt sollte insbesondere bei kritisch kranken Kindern berücksichtigt werden.

\section{》) Die "klinische Exomanalyse“ beschränkt sich auf bekannte Krankheitsgene}

Die Gesamtheit oben genannter Datenanalyse wird häufig als „klinische Exomanalyse" bezeichnet, da sie sich auf bekannte Krankheitsgene beschränkt. Finden sich hiermit keine Varianten, die ursächlich mit dem Phänotyp des Patienten in Zusammenhang gebracht werden können, folgt die Suche nach potenziell pathogenen Varianten in allen Genen. Erneut wird unter Zuhilfenahme genannter Datenbanken und Software-Programme nach seltenen, proteinverändernden Varianten gesucht.

Hierzu ein Beispiel: In der Trio-WES eines Kleinkinds mit der klinischen Diagnose eines Leigh-Syndroms (Laktatacidose, Epilepsie, bilaterale Basalganglienveränderungen im MRT) finden sich 2 seltene Funktionsverlustvarianten in ei- nem Gen, das für eine Untereinheit des mitochondrialen Komplexes I der Atmungskette codiert. Bisher sind allerdings noch keine Patienten mit Mutationen in diesem Gen beschrieben. Beide Elternteile sind je Träger einer Variante, womit eine Compound-Heterozygotie beim Kind bestätigt wird. Daraufhin folgt die funktionelle Bestätigung. In Fibroblasten des Patienten wird zunächst ein Komplex-I-Mangel nachgewiesen. Nachfolgend werden die Zellen mithilfe lentiviraler Transfektion mit der fehlerlosen (Normal-)Variante des betroffenen Gens ,ausgestattet", und es wird gezeigt, dass die Komplex-I-Aktivität wiederhergestellt wird. Hiermit ist sowohl die Pathogenität der Variante nachgewiesen als auch der Bezug zum Phänotyp hergestellt.

Häufig ist eine funktionelle Bestätigung nicht möglich, und der Nachweis der Pathogenität wird über die Tatsache geführt, dass sich mehrere Patienten mit sowohl dem gleichen Phänotyp als auch mit Varianten im gleichen Gen finden. Auch hier gibt es internetbasierte Plattformen (z. B. www.genematcher.org), die es Diagnostikern ermöglicht, miteinander in Kontakt zu treten und Daten auszutauschen, wenn beide Parteien Patienten mit Varianten in einem bisher nichtbeschriebenen Krankheitsgen gefunden haben.

\section{Diagnostische Ausbeute}

Durchschnittliche Raten klinisch-genetischer Diagnostik bei typischen pädiatrischen Indikationsstellungen sind in
- Tab. 3 zusammengefasst. Hierbei fällt auf, dass die diagnostische Ausbeute zwar im Vergleich zur Prä-NGS-Ära deutlich höher, allerdings weit entfernt von $100 \%$ ist. Hierbei spielen diverse Faktoren eine Rolle. Prinzipiell können Varianten in Genabschnitten liegen, welche durch WES nicht abgedeckt werden. Das betrifft generell Introne, je nach Sequenzierqualität ca. $2 \%$ aller Exone und z. B. Regionen, die reich an CpG-Dinukleotiden (Desoxycytidin, Phosphorsäure, Desoxyguanosin in $5^{\prime} \rightarrow 3^{\prime}$-Richtung) sind. Des Weiteren werden bestimmte Varianten (z.B. Trinukleotid-Repeats) mit der gängigen Sequenzier-Methode nicht detektiert; auch ist die Methode z. B. ungeeignet, die Kopienzahlbestimmungen der SMN1- und SMN2-Gene vorzunehmen, sodass eine vorliegende spinale Muskelatrophie (SMA) nicht diagnostiziert werden kann. Außerdem ist von ca. $70 \%$ der Gene des Menschen immer noch keine Funktion bekannt (oder ihnen wurde eine falsche Funktion zugeordnet), und Varianten können in diesen Genen falsch interpretiert werden. Eine jährliche Reanalyse der Daten ist daher sinnvoll [18]. Des Weiteren ist es schwierig, heterozygote Varianten als pathogen einstufen, weshalb es bei Verdacht auf Krankheiten (Entwicklungsverzögerung, Epilepsie), die häufig durch heterozygote de novo Varianten verursacht werden, sinnvoll ist, DNA von den Eltern-Kind-Trios zu sequenzieren. Eine weitere Herausforderung stellt dar, dass bei einer nichtgeringen Anzahl von Patienten mehrere monogene Krankhei- 
Tab. 3 Typische pädiatrischen Indikationsstellungen für genetische Diagnostik

Typische pädiatrisches Krankheitsbild Geeignete genetisch-diagnostische

\begin{tabular}{l|l|}
\hline Verminderte Sehfähigkeit $^{\mathrm{a}}$ & Gen-Panel \\
\hline Schwerhörigkeit/Taubheit & Gen-Panel \\
\hline Intelligenzminderung, isoliert & Trio-WES \\
\hline
\end{tabular}

Epileptische Enzephalopathie Trio-WES

Entwicklungsstörung Trio-WES

Kardiovaskuläre Erkrankungen Abhängig von Phänotyp (z. B. Karyogramm bei

Mitochondriale Erkrankungen (Trio-)WES

Vital bedrohter Säugling ( $<100$ Tage alt) Trio-WES

Klein-/Großwuchs

V.a. neurometabolische Erkrankung

Erhöhte Blutungsneigung

Kardiovaskuläre Erkrankungen

Immundefekt/Neutropenie

Metabolische Entgleisung (Intoxikationsbild/Hyperammonämie), Hypoglykämie

Akutes Leberversagen

Auffälliges Neugeborenenscreening

Intersexuelles Genitale

Angeborene Fehlbildungen, Dysmorphien, Fehlbildungen des Skeletts, der Organe etc.

${ }^{a}$ Einschließlich retinaler Veränderungen, Katarakt, Optikusatrophie etc.

$P C R$ "polymerase chain reaction", WES "whole exome sequencing", WGS "whole genome sequencing" Methode V.a. Trisomie 21, Array bei V.a. Mikrodeletionssyndrom, Trio-WES bei V.a. RASopathie wie z. B. Noonan-Syndrom)

Diagnoserate (Next-generation-sequencingMethode)

$51 \%$ (48\% Gen-Panel, zusätzlich 2\% WES bei negativem-Gene-Panel; [8])

$57 \%$ (30\% Einzelgenanalyse, 39\% WES; [6])

Kumulativ $42 \%$ (11,6\% Array, $24 \%$ WES nach negativem Array, $26 \%$ WGS nach negativem Array und negativer WES; [5])

$32,5 \%$ WES; [28]

$27 \%$ Array plus Trio-WES [32]

WES $8,4 \%$ [23]

$31 \%$ WES (eigene Daten)

$26 \%$ Karyotypisierung und/oder Einzelgenanalyse [16]; $37 \%$ WES, WGS, [17]; $57 \%$ WGS, [31]

$36 \%$ Trio-WES [7]

V.a. Turner-Syndrom, z. B. methylierungssensitive PCR bei V.a. Prader-Willi-Syndrom etc.)

WES

$68 \%$ WES [27]

Einzelgenanalyse gemäß Gerinnungsanalyse

Je nach Phänotyp Array (z. B. bei V.a. velokardiofaziales Syndrom oder Williams-BeurenSyndrom), (Trio)-WES bei Kardiomyopathie, Einzelgenanalyse bei V.a. Marfan-Syndrom

Abhängig vom Immunstatus, Gen-Panel oder (Trio)-WES

Einzelgenanalyse gemäß Metabolitenprofil

(Trio-)WES (nach Ausschluss infektiöser/ metabolischer Ursachen)

Einzelgenanalyse gemäß Metabolitenprofil bzw. endokrinologischer Befunde

Chromosomenanalyse

Abhängig von Phänotyp
$-$

$-$
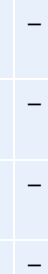

$-$ ten parallel und somit ein gemischter Phänotyp vorliegen [2].

\section{"Garbage in, garbage out"}

Die Kenntnis dieser technischen Limitationen ist sehr wichtig, nicht nur, um den richtigen ersten diagnostisch-genetischen Schritt zu setzen, aber auch um bei einer negativen WES den nachfolgenden diagnostischen Schritt auszuwählen. An dieser Stelle soll ebenfalls darauf hingewiesen sein, dass die Qualität der Befundung jedes diagnostischen Tests von der Qualität der Fragestellung und den zur Verfügung gestellten Informationen in der Anforderung abhängt. In der Informatik wurde hierfür der Begriff „garbage in, garbage out" geprägt.

Als letzter Aspekt soll nicht unerwähnt bleiben, dass betroffene Kinder nicht selbst in die Untersuchung einwilligen bzw. diese ablehnen können. Damit ergibt sich eine zusätzliche Verantwortung für den betreuenden Arzt und es sollte darüberhinaus bedacht werden, dass im Rahmen von NGSUntersuchungen nicht behandelbare, sich erst im höheren Erwachsenenalter manifestierende Erkrankungen als Nebendiagnosen auffallen können.

\section{Zusammenarbeit zwischen Pädiatrie und Humangenetik}

Das Wissen über die Herausforderungen und Limitierungen genomweiter Analysen und die korrekte Aufklärung des Patienten sind ein nicht zu unterschätzender Teil der genetischen Beratung und Diagnostik. Dies erfordert die enge Zusammenarbeit zwischen dem Fach- 


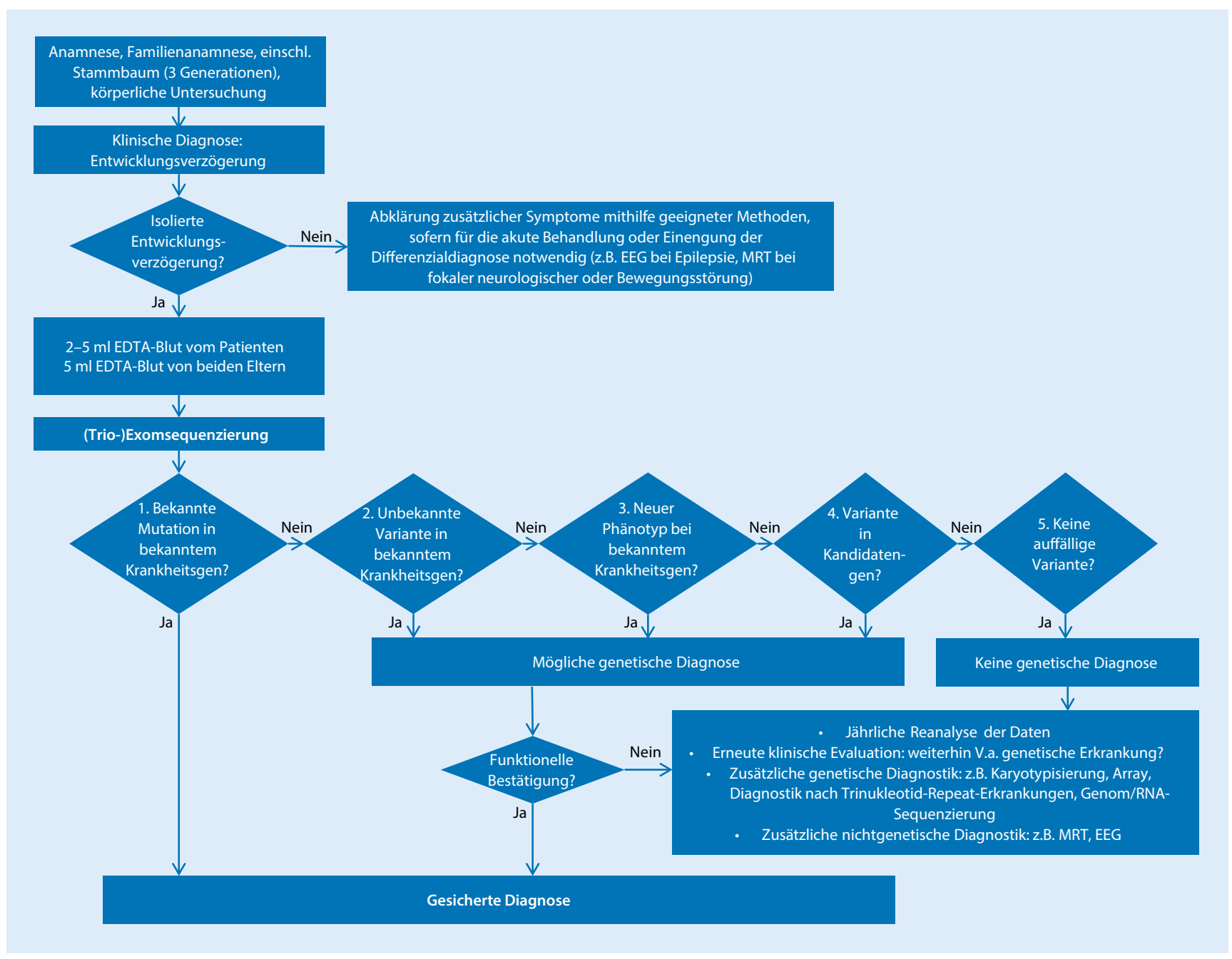

Abb. 2 - Flowchart zur Illustration des diagnostischen Vorgehens bei unklarer Entwicklungsverzögerung. EEG Elektroenzephalographie, MRT Magnetresonanztomographie

arzt für Kinder- und Jugendmedizin/ Jugendheilkunde, dem Facharzt für Humangenetik und dem genetischen Labor.

\section{Von der Diagnose zur Behandlung}

Die Diagnose ist das primäre Ziel eines jeden Arztbesuchs, erst darauf basierend kann die korrekte Behandlung erfolgen. Das klingt banal, ist aber auch in der „NGS-Ära“ immer noch richtig. Exomdiagnosen galten lange als teuer und wenig erfolgsversprechend. Verbesserte Sequenzmethoden, Auswerte-Programme und umfangreiche Datenbanken haben das Bild inzwischen geändert. Das Primat der Diagnose bei der Versorgung von Patienten mit dem Verdacht auf eine monogene Erkrankung steht außer Fra- ge. Eine korrekte, genetische Diagnose validiert den klinischen (biochemischen, radiologischen etc.) Phänotyp des Patienten und gibt ihr einen Namen. Für Eltern von Kindern mit Intelligenzminderung konnte gezeigt werden, dass eine Diagnose v.a. unter den folgenden Gesichtspunkten wichtig war. Die Diagnose bestätigte die Eltern darin, dass mit dem Kind „etwas nicht stimmt“. Sie fühlten sich ernst genommen und unterstützt beim Kampf für das Wohlergehen ihres Kindes. Eine Diagnose gleich welcher Art gab den Eltern sofort das Gefühl der Hoffnung für die Zukunft. Die Eltern gaben weiterhin an, dass die Diagnose half, die Erwartungen zu steuern. Die Bescheinigung einer Diagnose war außerdem notwendig im Umgang mit Behörden, z. B. zur Vorlage bei diversen Instanzen (Hilfs- mittel, Schulwahl). Die Eltern teilten mit, dass eine Diagnose sie darin unterstützte, Kontakt zu anderen betroffenen Eltern zu finden und dadurch Hilfe zu erfahren. Der Wille, „es einfach wissen zu wollen“, ebenso wie die Bedeutung der Diagnose für die pränatale Testung waren bei den befragten Familien sehr unterschiedlich ausgeprägt [15].

\section{》) Die genetische Diagnose validiert den klinischen Phänotyp des Patienten}

Letztendlich soll die Diagnose die Behandlung des Patienten verbessern und bestenfalls eine Therapie und Heilung bieten. Letzteres ist momentan noch selten, allerdings ergeben sich mit zuneh- 
mender Kenntnis genetischer Mechanismen bzw. der betroffenen Stoffwechselwege neue Behandlungsmöglichkeiten.

Beispielhaft ist die Behandlung der SMA mit dem Antisense-Oligonukleotid Nusinersen zu nennen. Dieses intrathekal $\mathrm{zu}$ verabreichende Medikament beeinflusst das "splicing“ des SMN2Gens und erhöht so indirekt die Menge an funktionsfähigem „survival motor neuron (SMN) protein“ im Liquor. Die Behandlung verbesserte bei $77 \%$ von 61 SMA-Typ-1-Patienten die motorischen Funktionen; Langzeitergebnisse müssen folgen, insbesondere auch hinsichtlich der Behandlung von Patienten mit anderen SMA-Typen und bezüglich der Kosten-Nutzen-Analyse [20]. Ein weiteres Beispiel ist die Behandlung von Patienten mit dem seltenen CAD-Defekt (epileptische Enzephalopathie, Poikilozytose), einer Störung in der Pyrimidinsynthese, bei der die Gabe von Uridin $\mathrm{zu}$ sofortiger Anfallsfreiheit und sogar zum Wiedererlangen von Entwicklungsrückschritten führte [12]. Vor diesem Hintergrund ist auch zu erwähnen, dass eine möglichst frühzeitige Diagnosestellung den Behandlungserfolg für viele Patienten essenziell beeinflussen dürfte [26].

In einigen Fällen ist die Diagnose wichtig, gerade um eine Therapie nicht durchzuführen (z. B. Lebertransplantation bei Nachweis eines mitochondrialen Depletionssyndroms mit progressiver neurologischer Beteiligung) oder generell bei der Entscheidung zur Therapielimitierung. Zusammenfassend kann der Einsatz von NGS-Techniken die „diagnostische Odyssee“ vieler pädiatrischer Patienten stark verkürzen und so auch vielen Kindern invasive Untersuchungen ersparen (z.B. Muskelbiopsie bei V.a. mitochondrialer Erkrankung). Letztendlich erspart diese auch dem behandelnden Team viel Zeit und kann zur Kosteneffizienz im Gesundheitssystem beitragen [19].

\section{Voraussetzungen für die erfolgreiche Diagnosestellung}

Diagnose und Behandlung von pädiatrischen Patienten mit genetischen Erkrankungen können nur im Team gelingen.
Voraussetzungen hierfür sind die gute Zusammenarbeit zwischen allen beteiligten Spezialisten ebenso wie der Zugang zu den diversen genetischen und nichtgenetischen Diagnosemethoden. Es ist ein enger Austausch zwischen dem niedergelassenen Facharzt für Kinder- und Jugendmedizin, der die Familie am besten kennt, dem spezialisierten Kinderfacharzt (z. B. Neuropädiater), dem Facharzt für Humangenetik und dem diagnostischen Labor notwendig, um alle diagnostischen und therapeutischen Möglichkeiten auszuschöpfen und keine Diagnosen $\mathrm{zu}$ verpassen.

Die hauptverantwortlich betreuende Ärzteschaft, die den genetischen Test anfordert, muss zunächst den Phänotyp und eine Verdachtsdiagnose definieren; hierzu sind eine ausführliche Anamnese, eine Erhebung der Familienanamnese, einschließlich eines Stammbaums über 3 Generationen, ebenso wie eine gründliche klinische Untersuchung essenziell. (Diesen Ablauf zeigt - Abb. 2 am Beispiel einer unklaren Entwicklungsverzögerung.) Als nächsten Schritt muss der behandelnde Arzt mögliche Differenzialdiagnosen einbeziehen. Basierend hierauf sollte die Methode ausgesucht werden, mit der am ehesten eine Diagnose erwartet werden bzw. eine signifikante Einengung der Differenzialdiagnosen erfolgen kann. Für einige wenige Erkrankungen wird diese eine Einzelgenanalyse sein, in den meisten Fällen die WES. Bei erfolgloser WES-Analyse sind Empfehlungen wichtig für den Zeitpunkt und die Art von Reanalysen. Eventuell gelingt die Rekrutierung im Rahmen eines Forschungsprogramms zur Entdeckung bisher unbekannter Gen-Phänotyp-Zusammenhänge. Bei erfolgreicher Diagnosestellung ist es wichtig, dass eine genetische Beratung über die erhobenen Befunde und ihre Konsequenz z. B. bezüglich der Familienplanung erfolgt. Auch ist es wichtig, dass sich die hauptbetreuenden Ärzte regelmäßig über Behandlungsmöglichkeiten (ebenfalls im Rahmen von Studien) informieren und diese mit der Familie besprechen. Die Versorgung von Patienten mit seltenen Erkrankungen sollte multidisziplinär und in enger Absprache mit den heimatna- hen Ärzten im Rahmen von Zentren für seltene Erkrankungen gebündelt werden.

\section{Zukunftsaussichten}

Die Technik der WES wird vermutlich auf Dauer von der WGS abgelöst werden, auch werden Integrative-multi-omicsAnsätze („metabolomics“, "proteomics“ etc.) genutzt werden. Das Neugeborenenscreening wird als genetisches Screening durchgeführt werden; die Daten werden lebenslang abrufbar sein. Der Patient wird sich je nach Lebenslage nach den relevanten Daten (z. B. Unverträglichkeit gewisser Chemotherapeutika bzw. Anästhetika, Risiko genetischer Erkrankungen bei Kinderwunsch etc.) erkundigen können.

\section{Fazit für die Praxis}

- Jedes Kind mit Verdacht auf eine genetische Erkrankung und seine Eltern sollten umfassend über die Möglichkeiten der genetischen Diagnostik beraten werden.

- Die neuen Sequenzierungsmethoden des "next generation sequencing" (Gen-Panel, "whole exome sequencing“, "whole genome sequencing") haben die molekulare Genetik und die Diagnostik in der Pädiatrie revolutioniert.

- Eine Diagnosestellung ist nur im Team aus niedergelassenen Fachärzten für Kinder- und Jugendmedizin/ Jugendheilkunde, den Spezialisten für das jeweilige pädiatrische Fachgebiet, Fachärzten für Humangenetik und genetischen Laborspezialisten möglich und sinnvoll.

- Aus einer genetisch basierten Diagnosestellung kann sich das Potenzial für eine gezielte therapeutische Intervention ergeben.

- Die Versorgung von Patienten mit seltenen Erkrankungen sollte multidisziplinär und in enger Absprache mit den heimatnahen Ärzten im Rahmen von Zentren für seltene Erkrankungen gebündelt werden. 


\section{Korrespondenzadresse}

PD Dr. med. S. B. Wortmann, PhD

Kinderklinik, Salzburger Landeskliniken (SALK) Müllner Hauptstr. 38, 5020 Salzburg, Österreich s.wortmann-hagemann@salk.at

Funding. Open access funding provided by Paracelsus Medical University.

\section{Einhaltung ethischer Richtlinien}

Interessenkonflikt. S.B. Wortmann und H.-C. Duba geben an, dass kein Interessenkonflikt besteht.

Dieser Beitrag beinhaltet keine von den Autoren durchgeführten Studien an Menschen oder Tieren.

Open Access. Dieser Artikel wird unter der Creative Commons Namensnennung 4.0 International Lizenz (http://creativecommons.org/licenses/by/4.0/deed. de) veröffentlicht, welche die Nutzung, Vervielfältigung, Bearbeitung, Verbreitung und Wiedergabe in jeglichem Medium und Format erlaubt, sofern Sie den/die ursprünglichen Autor(en) und die Quelle ordnungsgemäßnennen, einen Link zur Creative Commons Lizenz beifügen und angeben, ob Änderungen vorgenommen wurden.

\section{Literatur}

1. Adzhubei I, Jordan DM, Sunyaev SR (2013) Predicting functional effect of human missense mutations using PolyPhen-2. Curr Protoc Hum Genet. https:// doi.org/10.1002/0471142905.hg0720s76

2. Boycott KM, Innes AM (2017) When one diagnosis is not enough. N Engl J Med 376:83-85

3. De Braekeleer M, Dao TN (1990) Cytogenetic studies in couples experiencing repeated pregnancy losses. Hum Reprod 5:519-528

4. Dodge JA, Chigladze T, Donadieu J et al (2011) The importance of rare diseases: from the gene to society. Arch Dis Child 96:791-792

5. Gilissen C, Hehir-Kwa JY, Thung DT et al (2014) Genome sequencing identifies major causes of severe intellectual disability. Nature 511:344-347

6. Guan Q, Balciuniene J, Cao Ket al (2018) AUDIOME: a tiered exome sequencing-based comprehensive gene panel for the diagnosis of heterogeneous nonsyndromic sensorineural hearing loss. Genet Med.https://doi.org/10.1038/gim.2018.48

7. Guo MH, Shen Y, Walvoord EC et al (2014) Whole exome sequencing to identify genetic causes of short stature. Horm Res Paediatr 82:44-52

8. Haer-Wigman L, Van Zelst-Stams WA, Pfundt Retal (2017) Diagnostic exome sequencing in 266 Dutch patients with visual impairment. Eur J Hum Genet 25:591-599

9. Hempel M, Haack T, Eck S et al (2011) Monatsschr Kinderheilkd 159:827. https://doi.org/10.1007/ s00112-011-2446-y

10. Hoffman J (1995) Incidence of congenital heart disease: II. Prenatal incidence. Pediatr Cardiol 16:155-165

11. Kircher M, Witten DM, Jain P, O'Roak BJ, Cooper GM, Shendure J (2014) A general framework for estimating the relative pathogenicity of human genetic variants. Nat Genet46(3):310-315. https:// doi.org/10.1038/ng.2892

12. Koch J, Mayr JA, Alhaddad B et al (2017) CAD mutations and uridine-responsive epileptic encephalopathy. Brain 140:279-286

13. Kumar P, Henikoff S, Ng PC (2009) Predicting the effects of coding non-synonymous variants on protein function using the SIFT algorithm. Nat Protoc4(7):1073-1081

14. Macklon NS, Geraedts JP, Fauser BC (2002) Conception to ongoing pregnancy: the 'black box' of early pregnancy loss. Hum Reprod Update 8:333-343

15. Makela NL, Birch PH, Friedman JM et al (2009) Parental perceived value of a diagnosis for intellectual disability (ID): a qualitative comparison of families with and without a diagnosis for their child's ID. Am J Med Genet A 149a:2393-2402

16. Malam F, Hartley T, Gillespie MK et al (2017) Benchmarking outcomes in the neonatal intensive care unit: cytogenetic and molecular diagnostic rates in a retrospective cohort. Am J Med Genet A. https://doi.org/10.1002/ajmg.a.38250

17. Meng L, Pammi M, Saronwala A et al (2017) Use of exome sequencing for infants in intensive care units: ascertainment of severe single-gene disorders and effect on medical management. JAMA Pediatr 171:e173438

18. NambotS, Thevenon J, KuentzPetal (2017) Clinical whole-exome sequencing for the diagnosis of rare disorders with congenital anomalies and/or intellectual disability: substantial interest of prospective annual reanalysis. Genet Med. https:// doi.org/10.1038/gim.2017.162

19. Palmer EE, Schofield D, Shrestha R et al (2018) Integrating exome sequencing into a diagnostic pathway for epileptic encephalopathy: evidence of clinical utility and cost effectiveness. Mol Genet Genomic Med6:186-199

20. Pechmann A, Langer T, Schorling D et al (2018) Evaluation of children with SMA type 1 under treatment with Nusinersen within the expanded access program in Germany. J Neuromuscul Dis. https://doi.org/10.3233/JND-180315

21. Pfundt R, Del Rosario M, Vissers L et al (2017) Detection of clinically relevant copy-number variants by exome sequencing in a large cohort of genetic disorders. Genet Med 19:667-675

22. Rauch A, Wieczorek D, Graf E et al (2012) Range of genetic mutations associated with severe nonsyndromic sporadic intellectual disability: an exome sequencing study. Lancet 380:1674-1682

23. Sifrim A, Hitz MP, Wilsdon A et al (2016) Distinct genetic architectures for syndromic and nonsyndromic congenital heart defects identified by exome sequencing. Nat Genet 48:1060-1065

24. Speicher MR, Carter NP (2005) The new cytogenetics: blurring the boundaries with molecular biology. Nat Rev Genet 6:782-792

25. Stosser MB, Lindy AS, Butler E et al (2018) High frequency of mosaic pathogenic variants in genes causing epilepsy-related neurodevelopmental disorders. Genet Med 20:403-410

26. Tan TY, Dillon OJ, Stark Z et al (2017) Diagnostic impact and cost-effectiveness of whole-exome sequencing for ambulant children with suspected monogenic conditions. JAMA Pediatr 171:855-862

27. Tarailo-Graovac M (2016) Exome sequencing and the management of neurometabolic disorders. N Engl J Med 374(23):2246-2255. https://doi.org/ 10.1056/NEJMoa1515792

28. Thevenon J, Duffourd Y, Masurel-Paulet $A$ et al (2016) Diagnostic odyssey in severe neurodevelopmental disorders: toward clinical whole-exome sequencing as a first-line diagnostic test. Clin Genet 89:700-707

29. Vissers L, Van Nimwegen KJM, Schieving JH et al (2017) A clinical utility study of exome sequencing versus conventional genetic testing in pediatric neurology. Genet Med 19:1055-1063

30. Vissers LE, De Ligt J, Gilissen C et al (2010) A de novo paradigm for mental retardation. Nat Genet 42:1109-1112

31. Willig LK, Petrikin JE, Smith LD et al (2015) Whole-genome sequencing for identification of Mendelian disorders in critically ill infants: a retrospective analysis of diagnostic and clinical findings. Lancet Respir Med 3:377-387

32. Wright CF, Fitzgerald TW, Jones WD et al (2015) Genetic diagnosis of developmental disorders in the DDD study: a scalable analysis of genome-wide research data. Lancet 385:1305-1314 
Fachnachrichten - In eigener Sache

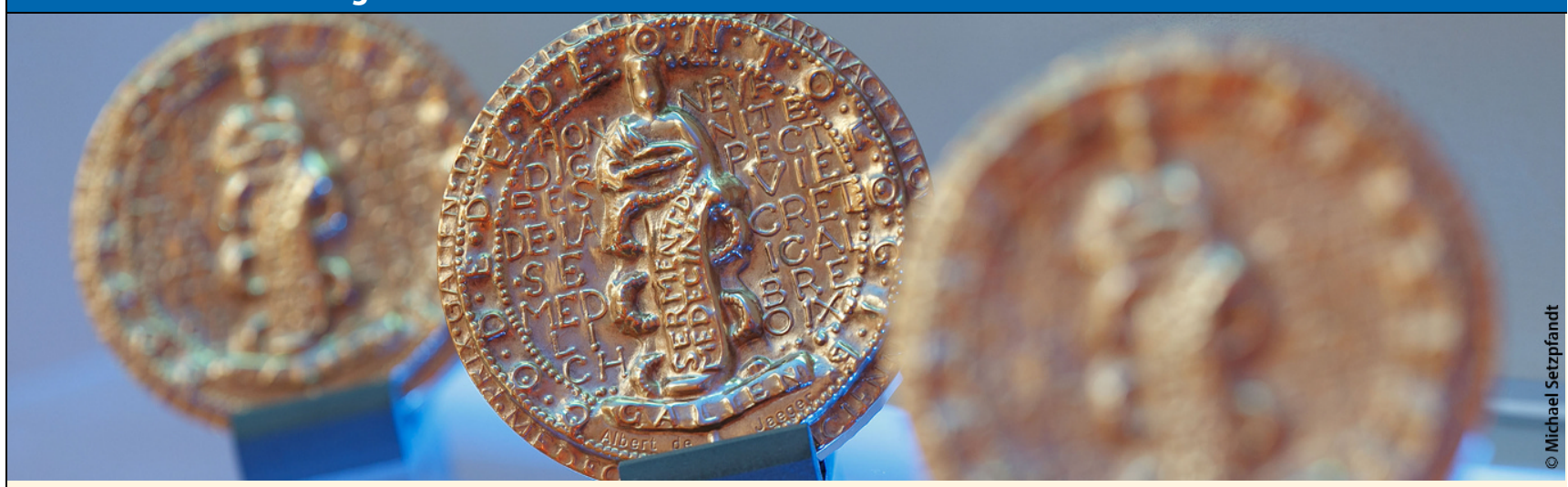

\section{Galenus-von-Pergamon-Preis 2018: 13 Arzneimittel sind im Rennen}

Mit dem von der Springer Medizin Verlag GmbH gestifteten Galenus-von-Pergamon-Preis werden seit 1985 in Deutschland herausragende Arzneimittelinnovationen gewürdigt. Der Preis wird in den Kategorien „Primary Care“, „Specialist Care“ und „Orphan Drugs“ vergeben. Über die Zuerkennung entscheidet eine unabhängige Expertenjury. Überreicht wird der Preis bei einem Festakt am 18. Oktober in Berlin. Hier stellen wir Ihnen drei Kandidaten vor:

\section{Spinraza $^{\circledR}$ (Nusinersen)}

In 95\% der Fälle liegen der spinalen Muskelatrophie (SMA) Mutationen auf Chromosom 5 zugrunde, die zu einem Mangel an dem für Motoneuronen lebenswichtigen Protein SMN (Survival of Motor Neuron) führen. Die Folge ist eine progrediente Degeneration von Motoneuronen im Rückenmark und Hirnstamm und eine schwere Muskelatrophie.

Die Erkrankung manifestiert sich häufig in den ersten sechs Lebensmonaten (infantile SMA). In der Regel lernen die betroffenen Kinder nie, selbstständig zu sitzen, und sterben ohne Therapie noch vor ihrem zweiten Geburtstag. Die Symptomatik der später einsetzenden SMA (later-onset SMA) ist zwar weniger schwer ausgeprägt, allerdings können bereits erworbene motorische Fähigkeiten im weiteren Verlauf der Erkrankung wieder verlorengehen. Mit der EU-Zulassung von Nusinersen (Spinraza ${ }^{\circledR}$ ) von Biogen zur Behandlung von Patienten mit $5 q$-assoziierter SMA ist erstmals eine gezielte Beeinflussung der Erkrankung möglich geworden. Nusinersen ist ein Antisense-Oligonukleotid, das die Produktion von funktionsfähigem SMN-Protein steigert. Der Gemeinsame Bundesausschuss (GBA) hat Nusinersen, das seit Juli 2017 in Deutschland erhältlich ist, einen „erheblichen“ Zusatznutzen für die infantile SMA (Typ I) attestiert.

\section{Hemlibra $^{\circledR}$ (Emicizumab)}

Hämophilie $A$ basiert auf einem genetisch bedingten Mangel oder einer funktionellen Beeinträchtigung des Gerinnungsfaktors VIII. Bisherige Therapien zielen auf die Substitution des fehlenden Faktors VIII. Etwa 30\% der Patienten entwickeln allerdings neutralisierende Antikörper (Hemmkörper) gegen den applizierten Gerinnungsfaktor VIII. Üblicherweise werden dann Bypass-Medikamente eingesetzt, entweder aktiviertes Prothrombinkomplex-Konzentrat oder rekombinanter aktivierter Faktor VII. Wegen der geringen Halbwertszeit müssen die Bypass-Medikamente bis zu mehrmals täglich als Infusionen i.v. appliziert werden.

Emicizumab (Hemlibra ${ }^{\circledR}$ ), von Roche und Chugai gemeinsam entwickelt, ist nach mehr als 20 Jahren die erste medikamentöse Therapieoption für Patienten mit Hämophilie und Hemmkörpern gegen Faktor VIII. Der bispezifische monoklonale Antikörper ist für die Routineprophylaxe von Blutungsereignissen bei Patienten aller Altersgruppen mit Hämophilie A und Faktor-VIII-Hemmkörpern zugelassen. Er wird in Deutschland seit Februar 2018 von Roche und Chugai vertrieben. Bei nur einmal wöchentlicher subkutaner Injektion ermöglicht die prophylaktische Behandlung mit Emicizumab einen effektiven Schutz vor Blutungsereignissen.

\section{Crysvita $^{\circledast}$ (Burosumab)}

Die angeborene X-chromosomale Hypophosphatämie (XLH) gehört zu den seltenen Erkrankungen und geht bei Kindern und Jugendlichen mit Wachstumsverzögerung und vielfältigen Knochendefekten einher. Im Erwachsenenalter dominieren Osteomalazie (Knochenschmerzen, Frakturen), Sehnenverkalkungen und Arthropathien infolge langjähriger Gelenkfehlstellung. Ursache der XLH ist ein Defekt im PHEX-Gen, der dominant vererbt wird oder durch spontane Mutation entsteht.

Mit dem rekombinanten humanen monoklonalen IgG1-Antikörper Burosumab (Crysvita ${ }^{\circledR}$ ) von Kyowa Kirin, der seit April 2018 in Deutschland erhältlich ist, kann jetzt erstmals auf kausale Weise in die Pathogenese der Erkrankung eingegriffen werden. Das subkutan anzuwendende Präparat ist zur Behandlung von Kindern ab einem Jahr und Jugendlichen in der Skelettwachstumsphase mit XLH und röntgenologischem Nachweis einer Knochenerkrankung zugelassen. Im Vergleich zur konventionellen Therapie mit mehrmals täglicher oraler Gabe von Phosphat und aktivem Vitamin D stellt die subkutane Antikörpertherapie nach den Studiendaten und den bisherigen Erfahrungen in der Praxis die effizientere und auch besser verträgliche Alternative dar.

Quelle: www.aerztezeitung.de 